ORIGINAL ARTICLE

\title{
Incidence of haematopoietic malignancies in US radiologic technologists
}

\author{
M S Linet, D M Freedman, A K Mohan, M M Doody, E Ron, K Mabuchi, B H Alexander, \\ A Sigurdson, M Hauptmann
}

Occup Environ Med 2005;62:861-867. doi: 10.1136/oem.2005.020826

See end of article for
authors' affiliations
$\ldots \ldots \ldots \ldots \ldots \ldots \ldots . . . \ldots$
Correspondence to:
Dr M S Linet, Radiation
Epidemiology Branch,
Division of Cancer
Epidemiology and
Genetics, National Cancer
Institute, 6120 Executive
Blvd. EPS Room 7054,
Bethesda, Maryland
20892, USA; linetm@
mail.nih.gov

Accepted 19 May 2005

\begin{abstract}
Background: There are limited data on risks of haematopoietic malignancies associated with protracted low-to-moderate dose radiation.

Aims: To contribute the first incidence risk estimates for haematopoietic malignancies in relation to work history, procedures, practices, and protective measures in a large population of mostly female medical radiation workers.

Methods: The investigators followed up 71894 (77.9\% female) US radiologic technologists, first certified during 1926-80, from completion of a baseline questionnaire (1983-89) to return of a second questionnaire (1994-98), diagnosis of a first cancer, death, or 31 August 1998 (731 306 person-years), whichever occurred first. Cox proportional hazards regression was used to compute risks.

Results: Relative risks (RR) for leukaemias other than chronic lymphocytic leukaemia (non-CLL, 41 cases) were increased among technologists working five or more years before 1950 ( $R R=6.6,95 \% \mathrm{Cl} 1.0$ to 41.9 , based on seven cases) or holding patients 50 or more times for $x$ ray examination $(R R=2.6,95 \% \mathrm{Cl}$ 1.3 to 5.4). Risks of non-CLL leukaemias were not significantly related to the number of years subjects worked in more recent periods, the year or age first worked, the total years worked, specific procedures or equipment used, or personal radiotherapy. Working as a radiologic technologist was not significantly linked with risk of multiple myeloma (28 cases), non-Hodgkin's lymphoma (118 cases), Hodgkin's lymphoma (31 cases), or chronic lymphocytic leukaemia (23 cases).

Conclusion: Similar to results for single acute dose and fractionated high dose radiation exposures, there was increased risk for non-CLL leukaemias decades after initial protracted radiation exposure that likely cumulated to low-to-moderate doses.
\end{abstract}

$\mathrm{R}$ adiation from a single high $\operatorname{dose}^{1}$ or fractionated radiotherapy ${ }^{2}$ is a well established risk factor for acute myeloid leukaemia (AML), and to a lesser extent for chronic myeloid and acute lymphoid leukaemia, but data are limited on risks of haematopoietic malignancies associated with protracted low-to-moderate dose exposures. Worldwide there are approximately 2.3 million medical radiation workers, $^{3}$ half of all workers exposed to manmade sources of radiation. Occupational studies reported increased leukaemia mortality among early radiologists, ${ }^{4-9}$ and increased risks in Chinese, ${ }^{10}$ but not Japanese, ${ }^{11}$ US Army, ${ }^{12}$ or Danish ${ }^{13}$ medical radiation workers. A statistically significant dose-response was apparent for AML in large cohorts of nuclear workers. ${ }^{14}{ }^{15}$ With the exception of the Danish medical radiation workers, few studies of medical or nuclear radiation workers have included substantial numbers of women. Findings have been inconsistent for multiple myeloma (MM) among the Japanese atomic bomb survivors, ${ }^{16}$ and radiation workers. ${ }^{4-15}$ Non-Hodgkin's lymphoma (NHL) and Hodgkin's lymphoma (HL) were not significantly increased in the atomic bomb survivors, ${ }^{1}$ patients treated with radiotherapy, ${ }^{2}$ or most radiation workers, ${ }^{3-7} 10-1517$ and radiation exposure has not been associated with increased risks of CLL. ${ }^{18}$ To contribute the first incidence risk information on haematopoietic malignancies in relation to work history, procedures, practices, and protective measures in a large population of mostly female medical radiation workers, we evaluated incidence risk for five categories (leukaemias other than CLL, MM, NHL, HL, CLL) of haematopoietic malignancies according to work history within the US Radiologic Technologists cohort.

\section{METHODS}

The methods for the cohort study of US radiologic technologists have been described in detail previously. ${ }^{19}$ Briefly, beginning in 1983, baseline questionnaires were mailed to 132454 (91\%) radiologic technologists located alive among 146022 certified nationwide during $1926-80$ by the American Registry of Radiologic Technologists. The questionnaire, which was completed and returned by 90305 respondents (68\% response rate), inquired about lifetime work history as a radiologic technologist, including procedures, practices, and protective measures. Lacking cumulative radiation dose estimates for individual technologists, the selfreported work history data were used to construct categorical proxy measures of radiation exposure. Other questions inquired about personal history of cancer, other serious diseases, and diagnostic and therapeutic radiation exposures; reproductive and family cancer history; and sociodemographic and lifestyle factors. During 1994-98, a second questionnaire was mailed to 126628 technologists located alive (96\% of the 132454 to whom the baseline questionnaire was mailed) to ascertain cancer occurrence and to collect detailed work, medical, and reproductive histories, and other cancer risk factor information. The research protocol for the cohort study of radiologic technologists was approved by the US National Cancer Institute's Special Studies Institutional Review Board and the University of Minnesota's Institutional

Abbreviations: AML, acute myeloid, monocytic, or myelomonocytic leukaemia; Cl, confidence interval; CLL, chronic lymphocytic leukaemia; CML, chronic myeloid leukaemia; HL, Hodgkin's lymphoma; MM, multiple myeloma; NHL, non-Hodgkin's lymphoma; RR, relative risk 


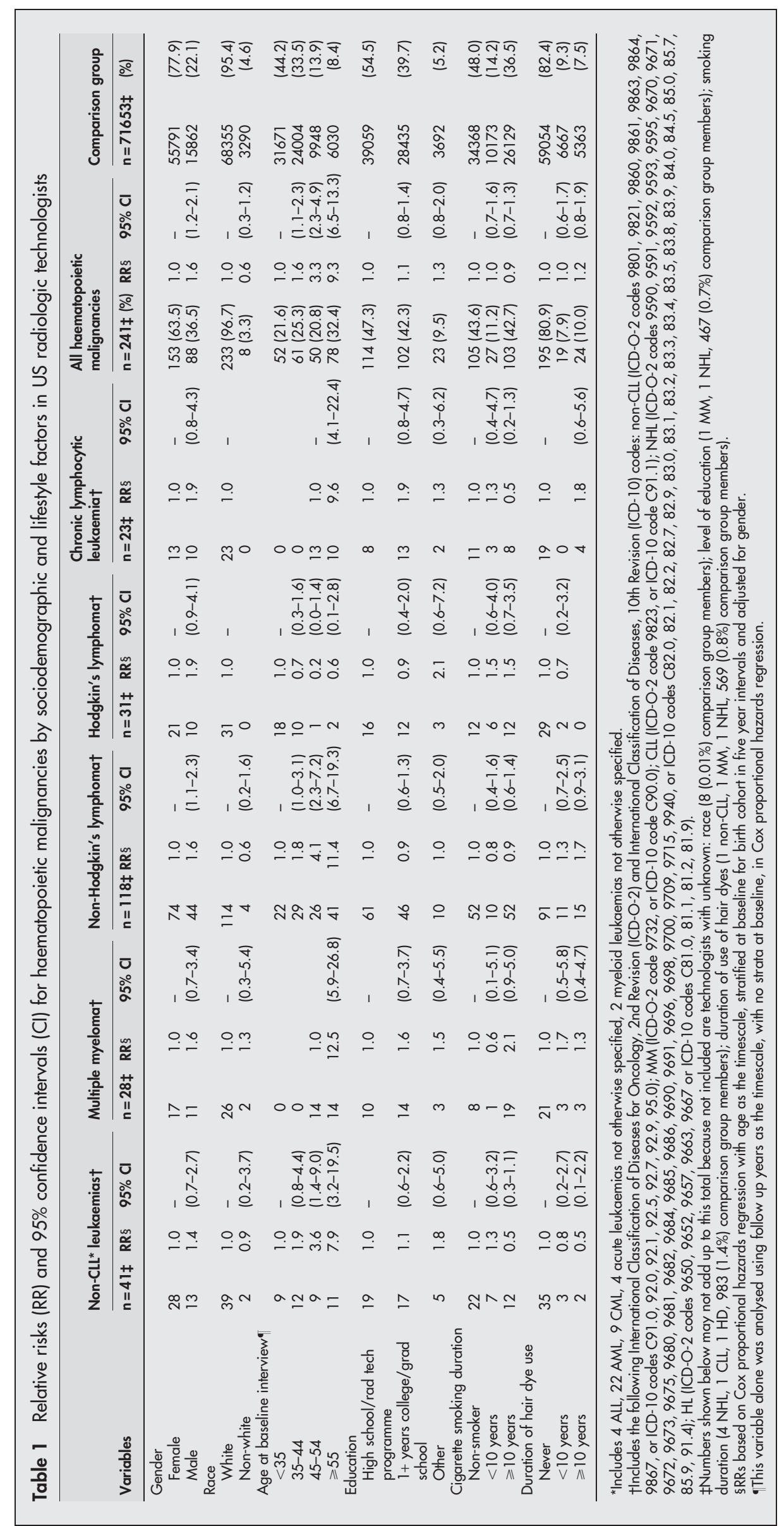




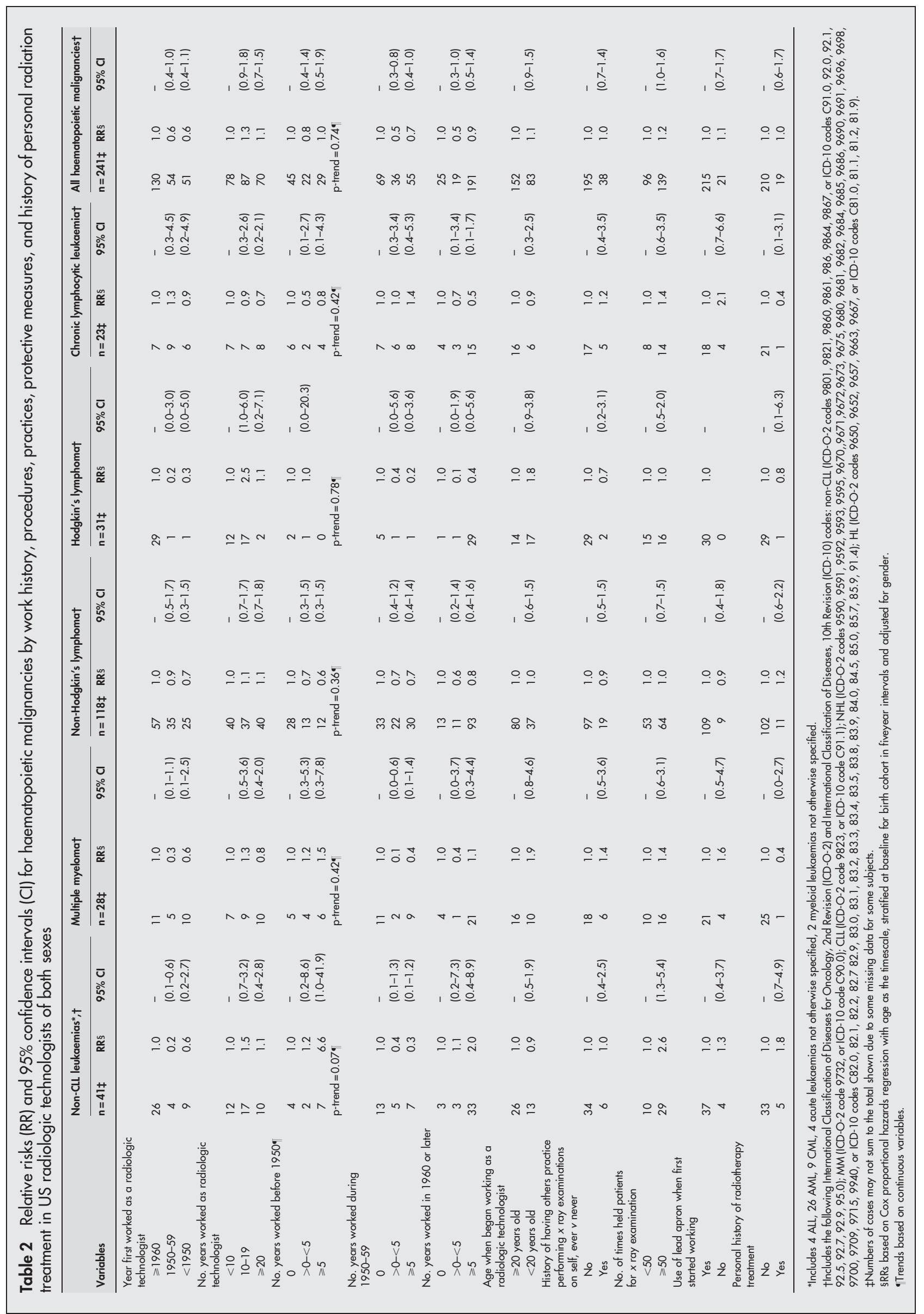


Review Board, and in accordance with an assurance filed with and approved by the US Department of Health and Human Services. Informed consent was obtained from each subject.

Of the 85372 baseline respondents who were still living when the second questionnaire was mailed, 70859 (83\%) completed the second questionnaire, including 69385 who were cancer-free (except for non-melanoma skin cancer). There were 2509 deaths ( 88 from haematopoietic malignancies and 2421 from other causes) before 31 August 1998 among those who completed the baseline questionnaire, but did not complete the second questionnaire.

Included in the study were 71 894 technologists who were cancer-free except for non-melanoma skin cancer, comprising 69385 technologists who completed both questionnaires plus 2509 technologists who completed the baseline questionnaire but died before completing the second questionnaire. Excluded were 16160 subjects who were cancer-free at completion of the baseline questionnaire, did not respond to the second questionnaire, but were not deceased as of 31 August 1998, since occurrence of haematopoietic malignancies was unknown in these technologists. Participants were followed from the return date of the baseline questionnaire until the return date of the second questionnaire, diagnosis of the first cancer (except non-melanoma skin cancer), death, or 31 August 1998, whichever occurred first.

From the questionnaire data and death certificate information, 241 study participants developed haematopoietic malignancies and 71653 did not develop haematopoietic malignancies. For validation, medical records were requested from hospitals and physicians, haematology and pathology reports were requested from laboratories for self-reported cases, and death certificates were requested for deceased cases. Eligible for this analysis were incident leukaemia cases with acute myeloid, acute monocytic, or acute myelomonocytic leukaemia (all combined designated as AML), acute lymphocytic leukaemia (ALL), chronic myeloid leukaemia (CML), acute leukaemia not otherwise specified, and other myeloid or unspecified myeloid leukaemia; all of these combined-that is, excluding chronic lymphocytic leukaemia (CLL), were designated non-CLL. Other primary haematopoietic malignancies evaluated were MM, NHL, HL, and CLL (see International Classification of Disease codes for the haematopoietic malignancies in table 1 footnotes). Of the 241 self-reported cases of haematopoietic malignancies, 205 $(85 \%)$ were confirmed by medical records or death certificates, including 95 of the 97 (98\%) self-reported cases for whom medical records were obtained, 22 erroneously selfreported as other malignancies but validated as having haematopoietic malignancies through review of the medical records requested for cancer validation, and 88 deaths ascribed to haematopoietic malignancies that were confirmed by death certificates. Since medical records confirmed $98 \%$ of the self-reported cases, 36 additional self-reported cases were included for which medical records could not be obtained.

Cox proportional hazards regression was used to compute relative risks (RR) and 95\% Wald confidence intervals (CIs), using age at diagnosis or death from haematopoietic malignancies as the response (that is, age as the timescale beginning at completion of the baseline questionnaire), and stratifying at baseline for birth cohort in five year intervals to control for secular trends. ${ }^{20}{ }^{21}$ Subjects were censored at the date of the first cancer other than a haematopoietic malignancy or non-melanoma skin cancer, the return date of the second questionnaire if no cancer was reported, or the date of death from a cause other than a haematopoietic malignancy. To assess risk by age at baseline questionnaire, calendar time was used as the time scale. Relative risks (RR) were assessed for each type of haematopoietic malignancy and all haematopoietic malignancies combined according to baseline questionnaire data on sociodemographic characteristics, cigarette smoking, and hair dye use, since these factors have been linked with one or more types of haematopoietic malignancies in some studies. ${ }^{22}$ Multivariate models were employed to assess potential confounding by these variables in analyses of job history, work practices, procedures performed, equipment used, and protective measures undertaken. ${ }^{20}$ Adjustment for duration of smoking or hair dye use did not alter the risk estimates for MM, NHL, or other haematopoietic malignancies associated with employment characteristics; thus, these variables were not included in the final statistical models. In tests for trend, we modelled exposures as continuous, and controlled for covariates. ${ }^{20}$

\section{RESULTS}

The 71653 radiologic technologists in the comparison group were mostly female $(77.9 \%)$, primarily Caucasian $(95.4 \%)$, and young at baseline (44.2\% were under age 35); a substantial proportion $(39.7 \%)$ had some college education (table 1). There were 731306 total person-years of follow up, on average 10.2 years per subject. Similar to haematopoietic malignancies in the general US population, ${ }^{22}$ cases were more likely to be male and older (except for HL) at baseline compared to the control group; more than half of the cases were age 45 or older at baseline, compared with $22 \%$ of the comparison group. There were no significant differences by race or education between haematopoietic malignancy cases and the comparison group (table 1), although there was a twofold increased risk for MM among technologists who smoked cigarettes for 10 or more years compared to nonsmokers, and a 70\% greater risk for NHL among those who used hair colouring products for a decade or more compared to never users (table 1 ).

The 41 non-CLL leukaemia cases included 22 AML, nine CML, four ALL, four incompletely specified as acute leukaemias, and two incompletely specified as myeloid leukaemias. Risk for non-CLL leukaemias rose (ptrend $=0.07$ ) with increasing years worked as a technologist before 1950, and was increased nearly sevenfold (based on seven cases) among those working five or more years during this period (table 2). Technologists who held patients 50 or more times during $x$ ray examinations experienced a 2.6-fold significantly increased risk of non-CLL leukaemia. We found similar results when we narrowed the case group to AML $(\mathrm{n}=22)$ (e.g. $\mathrm{RR}=2.5$, 95\% CI 0.3 to 21.5 among technologists who worked five or more years before 1950; RR $=2.4$, $95 \%$ CI 0.9 to 6.3 among technologists who held patients 50 or more times), and when we assessed non-CLL among the smaller group of male technologists $(\mathrm{RR}=30.1$, 95\% CI 0.8 to 1103 for technologists who worked five or more years before 1950; $R R=2.1,95 \%$ CI 0.6 to 7.7 for technologists who held patients 50 or more times).

There were no significant excesses of non-CLL in analyses evaluating the year or age subjects first worked as radiologic technologists, the total number of years worked, or working for an increasing number of years during 1950-59 or in more recent time periods. The twofold increased risk for non-CLL among technologists who worked five or more years in 1960 or later was unexpected, but confidence limits were wide (table 2). No significant excesses of non-CLL were observed among radiologic technologists on whom others practiced performing $x$ ray examinations during training, or who did not use lead aprons at initial employment. Among the few radiologic technologists reporting a personal history of radiotherapy for benign conditions, the risks of non-CLL were $80 \%$, albeit non-significantly, increased (table 2). Risks for non-CLL were not linked with performing fluoroscopy, multi-film procedures, routine or dental $x$ ray examinations, 
computed tomography scans, radiotherapy procedures, or with using specific equipment (including orthovoltage, cobalt-60, betatron, or linear accelerator machines), or using radium or other radioisotopes (numbers were small) during any of the time periods (data not shown).

For the other haematopoietic malignancies, including CLL, there were no significant associations with any aspects of work history, procedures, practices, or protective measures (table 2). Unlike the findings for non-CLL, risks for the other haematopoietic malignancies were not significantly associated with working five or more years before 1950 or holding patients 50 or more times. As with non-CLL, there were no relations for other haematopoietic malignancies with any use or first working with fluoroscopic or multi-film procedures before 1950, or working with other specific radiologic procedures, particular equipment, or radioisotopes (data not shown). However, modest, non-significant $40-90 \%$ increases of MM were observed among radiologic technologists working five or more years before 1950, those first employed before age 20, those on whom others practiced performing $x$ ray examinations during training, those who held patients 50 or more times, or those who did not use a lead apron when first working as radiologic technologists.

\section{DISCUSSION}

In this follow up of US radiologic technologists, excesses of non-CLL leukaemias (mostly AML) occurred among technologists who worked five or more years before 1950 or those who reported holding patients 50 or more times during $x$ ray examinations. Non-CLL leukaemias were not associated with other work history characteristics. There were modest, albeit non-significant increases in risk of MM among radiologic technologists who worked five or more years before 1950, and engaged in work practices that might be associated with increased protracted radiation exposures. There were no excesses of non-Hodgkin's lymphoma, Hodgkin's lymphoma, or chronic lymphocytic leukaemia associated with the work history, specific practices, or lack of protective measures of the radiologic technologists.

The excess risks of leukaemia among the early workers likely reflected substantial radiation exposures from the high allowable exposures during the first half of the 20th century. Following the discovery of $x$ rays in 1895, recognition of radiation related dermatitis, severe tissue damage, and carcinogenesis led the US Advisory Committee on X-ray and Radium Protection to propose the first formal radiation protection standard of 0.1 roentgen per day (approximately $0.3 \mathrm{~Sv}$ per year, which typically included 300 work days) in $1934 .^{23}{ }^{24}$ Despite the standard, technologists continued to be exposed to levels as high as 100 roentgens ( $1 \mathrm{~Sv}$ ) per year in some workplaces. $^{25}$ A 1940 survey reported average and maximum daily exposure of 0.005 roentgen and considerably more than 0.1 roentgen, respectively; the latter often related to bedside radiography and holding patients. ${ }^{26}$ The International Commission of Radiological Protection proposed reducing the limits to $15 \mathrm{rem} /$ year (or $0.15 \mathrm{~Sv}$ per year bone marrow dose) in 1949, to $5 \mathrm{rem} /$ year (0.05 Sv per year) in 1957, and to the current limit of $0.02 \mathrm{~Sv}$ per year averaged over five years, not to exceed $0.05 \mathrm{~Sv}$ in any single year, in $1990 . .^{23}{ }^{27-30}$ Yet, weekly exposures in the 1950s were above the maximum permissible dose for $20 \%$ of radiologic technologists as measured in one survey, ${ }^{27}$ ranging as high as 0.3 roentgen per day (for example, $0.9 \mathrm{~Sv}$ per year). ${ }^{28}$ Film-badge monitoring was initiated in the late 1940s, and more widely adopted in the 1960s or later. ${ }^{9-11} 13$

The excess risks of incident non-CLL among the US radiologic technologists who worked five or more years before 1950 are based on only seven cases, but are consistent with the increased risks observed in early $\mathrm{UK}^{7}$ and US radiologists, ${ }^{8}$ and in the Chinese medical radiation workers who worked before $1970 .{ }^{10}$ Increased non-CLL risks have been reported in populations with widely varying radiation exposures, ranging from the moderate-to-high dose acute single exposures in Japanese atomic bomb survivors, ${ }^{16}$ to the very low-dose, low-dose rate radiation exposures in large populations of nuclear workers. ${ }^{14}{ }^{15}$ No significant excesses of leukaemia occurred in UK radiologists after $1920,{ }^{717}$ US radiologists after $1939,{ }^{89}$ US army ${ }^{12}$ or Japanese ${ }^{11}$ radiologic technologists, or in Danish ${ }^{13}$ radiotherapy department staff. In the present study, leukaemia risks were not increased among US radiologic technologists who first worked before age 20 , but the estimate was based on only 13 non-CLL cases. In contrast, leukaemia incidence risks were notably increased among the Chinese medical radiation workers who first worked before ages $20-25,{ }^{10}$ and the Japanese atomic bomb survivors exposed at young ages. ${ }^{1}$ Few radiation exposed cohorts have been followed for decades after initial exposure. ${ }^{1}{ }^{17}$ 30-32 $^{32}$ Follow up of this cohort of radiologic technologists did not commence until the baseline survey in 198389; hence, it is not possible to ascertain the pattern of the dose-response curve for non-CLL incidence before the early 1980s. Nevertheless, the apparent persistence of increased risks for non-CLL through the 1990s among the technologists who worked five or more years before 1950 is consistent with excess leukaemia mortality continuing for 20 years or more after certification in the post-1920 cohort of UK radiologists ${ }^{17}$ and after radiotherapy among patients with ankylosing spondylitis; ${ }^{31}$ both populations had protracted radiation exposures. Similarly, non-CLL risk remained increased for several decades after exposure among the Japanese atomic bomb survivors, despite the difference in the type of radiation exposure (that is, a single acute high dose rate exposure). ${ }^{1}$ Among male and female atomic bomb survivors exposed to the bombings in childhood or adolescence, risks of non-CLL subsequently rose, reaching highest absolute excess risk five to ten years after exposure, and thereafter declining rapidly with time in males, but more slowly with time in females. Among male and female Japanese atomic bomb survivors exposed at older ages, risks of non-CLL also increased within a few years after the bombings, peaking but attaining lower absolute excesses at five to ten years after exposure; subsequently risks declined with time after exposure, although the risk for women declined less rapidly than the risk for men. ${ }^{1}$

The results for non-CLL incidence among the US radiologic technologists are also consistent with the findings from an earlier analysis of mortality in this population during 1926$97 .{ }^{33}$ In both analyses, there were increased risks for non-CLL associated with working five or more years before 1950 and with holding patients 50 or more times. However, in the earlier mortality study, but not the current incidence investigation, there were increased risks for non-CLL among subjects who first worked as technologists or first worked with fluoroscopy or multi-film procedures prior to 1950. The differences were likely due to small numbers as well as some differences in the specific technologists in the two case groups; for example, cases included in the incidence analysis overlapped but differed in part from cases included in the mortality analysis.

In the current analysis, small non-significant incidence increases for multiple myeloma were associated with younger age when first employed, working five or more years prior to 1950, and several work practices. These findings are consistent with both the increased mortality risk seen for MM among $\mathrm{UK}^{71}$ and US radiologists ${ }^{8}{ }^{9}$ in the 1930s and 1940s, but not with the absence of excess MM in the US Army, ${ }^{12}$ Danish, ${ }^{13}$ Chinese, ${ }^{10}$ and Japanese ${ }^{11}$ medical radiation workers. The lack of increased MM among the Chinese and 
Japanese medical radiation workers is not surprising, given the low incidence of B-cell malignancies occurring in Asians. ${ }^{22}$ While there appeared to be an excess risk of MM mortality among the Japanese atomic bomb survivors, ${ }^{16}$ an analysis of incidence risks ${ }^{1}$ did not substantiate the earlier findings. US radiologists experienced increased mortality from lymphoma in the 1930-39 and the 1940-49 cohorts, but not in earlier cohorts, ${ }^{8}$ similar to the increase in lymphoma in the post-1920 cohort of UK radiologists. ${ }^{77}$ The lack of excess risks of lymphoma among the earliest radiologists, who likely had the highest levels of exposure among the US and British radiologists, ${ }^{7-9} 17$ suggests that these malignancies are not associated with radiation. There were no excesses of lymphoma (not evaluated separately for NHL versus HL) nor of CLL in the US Army, ${ }^{12}$ Danish, ${ }^{13}$ Chinese, $^{10}$ or Japanese ${ }^{11}$ medical radiation workers.

Strengths of the investigation of incidence risks among the US radiologic technologists compared with other studies of medical radiation workers include the notably larger size of the former, the female predominance, and the detailed individual work history information that was used as a proxy measure for radiation exposure. Other advantages of the study of US radiologic technologists were the high level of diagnostic confirmation and the focus on incidence, which is important for evaluating risks for indolent haematopoietic malignancies such as CLL, or those with relatively good survival rates such as HL and certain types of NHL. The main limitation is the absence of dosimetry data for individual technologists. In addition, the small number of non-CLL and MM cases precluded stable assessment of the temporal pattern of risk, particularly according to age at initial occupational radiation exposure (although relatively few technologists were first exposed to occupational radiation before age 18), or of changes in risk by sex, or leukaemia subtype; leukaemia risk patterns have been shown to vary among subgroups of these factors. ${ }^{30-32}$ The surrogate radiation exposure measures, namely work histories and practices, may not have captured some key determinants of individual differences in radiation exposure. The detailed assessment of incidence risks for the major categories of haematopoietic malignancies according to job history, procedures performed, and protective practices provides more radiation exposure related information than in previous reports focusing on cancer risks in medical radiation workers.

It is important to continue monitoring the US radiologic technologists, a cohort with a mean age of 53 currently, since there are few other studies internationally of medical radiation workers, and only a small subset of these studies followed the workers for long periods of time. A second important reason to continue monitoring is because of rapidly increasing low-to-moderate dose, fractionated radiation exposure to the general public associated with newer diagnostic (for example, spiral computed tomography or whole body scans, particularly because repeated examinations are often performed $)^{34}$ and interventional radiologic procedures (for example, those involving real-time fluoroscopy). ${ }^{35}$ As the numbers and types of newer radiological procedures characterised by substantial radiation doses have increased, public concern has grown about possible carcinogenic effects. Finally, most information about risks of haematopoietic malignancies from occupational exposures to ionising radiation comes from epidemiological studies of primarily male medical radiation or nuclear workers. In a population that included $78 \%$ female medical radiation workers, there were no significantly increased risks for NHL, HD, MM or CLL; however, radiologic technologists who experienced protracted fractionated radiation that likely cumulated to low-to-moderate doses developed excess nonCLL that occurred decades after initial radiation exposures, similar to earlier results for single acute dose and fractionated high dose exposure.

\section{ACKNOWLEDGEMENTS}

We are grateful to the radiologic technologists who participated in the cohort study of US Radiologic Technologists; Mr J Reid of the American Registry of Radiologic Technologists for continued support of this project; Ms D Kampa of the University of Minnesota for supervision of data collection and coordination; Ms K Chimes of Westat for data management; Mr R Van Dusen and Ms L Bowen of Information Management Services, Inc. for biomedical computing; and to Dr Genevieve Matanoski for helpful advice. We also acknowledge our appreciation to Drs J Boice, Jr and J Mandel who played a critical role in the initiation, design, and maintenance of this cohort study for many years. This work was supported in part by contracts NO1-CP-15673, NO1-CP-51016, NO2-CP-81005, NO2-CP81121 by the National Cancer Institute, National Institutes of Health, and US Public Health Services.

\section{Authors' affiliations}

M S Linet, D M Freedman, M M Doody, E Ron, K Mabuchi, A Sigurdson, M Hauptmann, Division of Cancer Epidemiology and Genetics, National Cancer Institute, Department of Health and Human Services, Bethesda, $M D$, USA

A K Mohan, Office of Biostatistics and Epidemiology, Center for Biologics Evaluation and Research, Food and Drug Administration, Rockville, MD, USA

B H Alexander, Division of Occupational and Environmental Health, University of Minnesota, Minneapolis, MN, USA

Funding: National Cancer Institute, National Institutes of Health, Department of Health and Human Services; contract numbers NO1CP-51016, NO2-CP-81121, NO2-CP-81005

Competing interests: none declared

\section{REFERENCES}

1 Preston DL, Kusumi S, Tomonaga M, et al. Cancer incidence in atomic bomb survivors. Part III. Leukemia, lymphoma and multiple myeloma, 1950-1987. Radiat Res 1994; 137:S68-97.

2 Inskip PD. Second cancers following radiotherapy. In: Neugut Al, Meadows AT, eds. Multiple primary cancers. Philadelphia, PA: Lippincott Williams \& Wilkins, 1999:91-135.

3 UNSCEAR (United Nations Scientific Committee on the Effects of Atomic Radiation). Sources and effects of ionizing radiation. United Nations Publication No. EOOIX.3. Annex I. Epidemiological evaluation of radiationinduced cancer. New York, NY: United Nations Scientific Committee on the Effects of Atomic Radiation, 2000:297-431.

4 March HC. Leukemia in radiologists. Radiology 1944;43:275-8.

5 Ulrich $\mathbf{H}$. The incidence of leukemia in radiologists. N Engl J Med 1946;234:45-6.

6 Lewis EB. Leukemia, multiple myeloma, and aplastic anemia in American radiologists. Science 1963;142:1492-4.

7 Smith PG, Doll R. Mortality from cancer and all causes among British radiologists. Br J Radiol 1981;54:187-94.

8 Matanoski GM, Seltser R, Sartwell PE, et al. The current mortality rates of radiologists and other physician specialists: specific causes of death. Am J Epidemiol 1975;101:199-210.

9 Matanoski GM, Sartwell P, Elliott E, et al. Cancer risks in radiologists and radiation workers. In: Boice JD Jr, Fraumeni JF Jr, eds. Radiation carcinogenesis: epidemiology and biological significance. New York, NY: Raven Press, 1984:83-96.

10 Wang JX, Zhang LA, Li BX, et al. Cancer incidence and risk estimation among medical X-ray workers in China, 1950-1995. Health Phys 2002;82:455-66.

11 Yoshinaga S, Aoyama T, Yoshimoto Y, et al. Cancer mortality among radiological technologists in Japan: updated analysis of follow-up data from 1969-1993. J Epidemiol 1999;9:61-72.

12 Jablon S, Miller RW. Army technologists: 29-year follow-up for cause of death. Radiology 1978;126:677-9.

13 Andersson M, Engholm G, Ennow K, et al. Cancer risk among staff at two radiotherapy departments in Denmark. Br J Radiol 1991;64:455-60.

14 Cardis E, Gilbert ES, Carpenter L, et al. Effects of low doses and low dose rates of external ionizing radiation: cancer mortality among nuclear industry workers in three countries. Radiat Res 1995; 142:117-32

15 Muirhead CR, Goodill AA, Haylock RG, et al. Occupational radiation exposure and mortality: second analysis of the National Registry for Radiation Workers. J Radiol Prot 1999;19:3-26.

16 ShimizuY, Schull WJ, Kato H. Cancer risk among atomic bomb survivors. The RERF Life Span Study. JAMA 1990;264:601-4.

17 Berrington A, Darby SC, Weiss HA, et al. 100 years of observation on British radiologists: mortality from cancer and other causes 1887-1997. Br J Radiol 2001;74:507-19. 
18 Sgambati M, Devesa SS, Linet MS. Epidemiology of chronic lymphocytic leukemia. In: Cheson BD, ed. Chronic lymphocytic leukemia: scientific advances and clinical developments. New York, NY: Marcel Dekker, 2001:33-62

19 Boice JD Jr, Mandel JS, Doody MM, et al. A health survey of radiological technologists. Cancer 1992;69:586-98.

20 Breslow NB, Day NE. Statistical methods in cancer research: the design and analysis of cohort studies, vol II. Lyon, France: IARC, 1987.

21 Korn EL, Graubard BI, Midthune D. Time-to-event analysis of longitudinal follow-up of a survey: choice of the time scale. Am J Epidemiol 1997; 145:72-80.

22 Linet MS, Devesa SS, Morgan GJ. New perspectives on the epidemiology of hematologic malignancies and related disorders. In: Shields PG, ed. Cancer risk assessment. New York, NY: Taylor \& Francis Group, 2005:671-716.

23 Inkret WC, Meinhold CB, Taschner JC. Protection standards: radiation and risk-a hard look at the data. Los Alamos Science 1995;23:117-24.

24 Miller RW. Delayed effects of external radiation exposures: a brief history. Radiat Res 1995; 144:160-9.

25 Braestrap CB. Past and present radiation exposure to radiologists from the point of view of life expectancy. Am J Roentgenol 1957;78:988-92.

26 Cowie DB, Scheele LA. A survey of radiation protection in hospitals. J Natl Cancer Inst 1941;1:767-87.
27 Spalding CK, de Amicis E, Cowing RF. Radiation-exposure survey of x-ray and isotope personnel. Nucleonics 1949;5:63-6.

28 Geist RM Glasser O, Hughes CR. Radiation exposure survey of personnel at the Cleveland Clinic Foundation. Radiology 1953:60:186-91.

29 Kumazawa S, Nelson DR, Richardson ACB. Occupational exposure to ionizing radiation in the United States: a comprehensive review for the year 1980 and a summary of trends for the years 1960-1985, EPA/520/1-8005. Washington, DC: US Government Printing Office, 1984

30 International Commission on Radiological Protection. 1990 recommendations of the International Commission on Radiological Protection. Publication 60. Oxford: Pergamon Press, 1991.

31 Weiss HA, Darby SC, Fearn T, et al. Leukemia mortality after x-ray treatment for ankylosing spondylitis. Radiat Res 1995;142:1-11.

32 Little MP, Weiss HA, Boice JD Jr, et al. Risks of leukemia in Japanese atomic bomb survivors, in women treated for cervical cancer, and in patients treated for ankylosing spondylitis. Radiat Res 1999;152:280-92.

33 Mohan AK, Hauptmann M, Freedman DM, et al. Cancer and other causes of mortality among radiologic technologists in the United States. Int J Cancer 2003;103:259-67.

34 Mettler FA Jr, Wiest PW, Locken JA, et al. CT scanning: patterns of use and dose. J Radiol Prot 2000;20:353-9.

35 Mettler FA Jr, Koenig TR, Wagner LK, et al. Radiation injuries after fluoroscopic procedures. Sem Ultrasound CT MRI 2002;23:428-42.

\section{1 th European Forum on Quality Improvement in Health Care}

26-28 April 2006, Prague, Czech Republic

For further information please go to: www.quality.bmipg.com

Book early to benefit from a discounted delegate rate 